Luanne Alves Oliveira ${ }^{1}$

Leonardo Rodrigo Baldaçara ${ }^{1}$

Maria Zoreide Brito Maia ${ }^{1}$
${ }^{1}$ Universidade Federal do Tocantins, Programa de Pós-graduação em Ciências da Saúde. Palmas, TO, Brasil.

Contato:

Luanne Alves Oliveira

E-mail:

luanne.siass@uft.edu.br

Trabalho baseado em dissertação de Luanne Alves Oliveira intitulada "Afastamentos por transtornos mentais entre servidores públicos federais no Tocantins", defendida em 2013 no Mestrado Profissional em Ciências da Saúde da Universidade Federal do Tocantins.

Trabalho não apresentado em reuniões científicas.

Os autores declaram não haver conflitos de interesses e que o trabalho não foi subvencionado.

\section{Afastamentos por transtornos mentais entre servidores públicos federais no Tocantins}

\author{
Absence from work due to mental disorders among federal \\ government workers in Tocantins, Brazil
}

\begin{abstract}
Resumo
Objetivo: analisar as características epidemiológicas de afastamentos do trabalho por Transtornos Mentais e de Comportamento (TMC) entre servidores públicos federais do Poder Executivo, no Tocantins, e investigar se variáveis pré-selecionadas influenciaram nos afastamentos. Métodos: foram avaliados dados de 103 servidores com pelo menos um registro de afastamento por transtornos mentais no período de abril de 2011 a dezembro de 2012. Os dados foram obtidos de prontuários eletrônicos e de informações do Portal da Transparência do Governo Federal Resultados: "Episódios depressivos", "Reações ao stress grave e transtornos de adaptação", e "Outros transtornos ansiosos" foram os mais frequentes causadores dos afastamentos. Diagnósticos de "Esquizofrenia, transtornos esquizotípicos e delirantes" apresentaram a maior média de afastamentos por servidor; os diagnósticos de "Transtornos devido ao uso de substância psicoativa" apresentaram a maior média de dias de afastamento por servidor. Na análise de correlação parcial, controlado o efeito da idade, os servidores com menos tempo de serviço público e de trabalho em órgão federal passaram mais dias afastados do trabalho do que aqueles que já trabalhavam nesses ambientes há mais tempo. Conclusão: os resultados reforçam a concepção de saúde mental e de trabalho como indissociáveis na conjuntura produtiva atual, incluindo o serviço público.
\end{abstract}

Palavras-chave: transtornos mentais; saúde do trabalhador; setor público.

Keywords: mental disorders; occupational health; public sector. 


\section{Introdução}

O aumento do estresse relacionado ao trabalho vem sendo destacado em alguns estudos como uma das importantes causas de Transtornos Mentais, acarretando também no aumento do absenteísmo. Tais estudos evidenciam o aumento no número de pessoas que adoecem e se afastam do trabalho pelos mais variados motivos de saúde, contudo os Transtornos Mentais e de Comportamento (TMC) têm sido os mais prevalentes e são uma das maiores causas de afastamento do trabalho de longo prazo (CUNHA, 2008; SELIGMANN-SILVA, 2009).

Os TMC são condições clinicamente significativas com características de alterações de humor, do modo de pensar ou de comportamentos associados com angústia pessoal e/ou deterioração do funcionamento, de acordo com a Organização Mundial da Saúde (2001).

De acordo com dados do Ministério do Planejamento, Orçamento e Gestão (MPOG) publicados em 2005, os gastos com aposentadorias precoces e licenças de afastamento do trabalho foram estimados em R\$ 300 milhões ao ano (BRASIL, 2005). À época, a proporção de aposentadorias precoces e de afastamentos do trabalho no serviço público federal chegava a $14 \%$, enquanto o índice no setor privado era de $2 \%$ dos trabalhadores (BRASIL, 2005). As três principais causas dos afastamentos eram: lesão por esforço repetitivo - LER (35\%), depressão (13\%) e dependência química (7\%) (BRASIL, 2005). Dados mais recentes do Ministério da Previdência Social mostram que, em nível nacional, de janeiro a maio de 2013, 5.315 auxílios-doença acidentários foram concedidos a contribuintes devido aos TMC (BRASIL, 2013a). Dentre esses, 786 foram concedidos àqueles vinculados à administração pública em geral, um quantitativo que representa $14,78 \%$ dos auxílios-doença acidentários concedidos pela mesma razão ao total de contribuintes (BRASIL, 2013b).

No Brasil, o servidor público, apesar de possuir certa estabilidade no trabalho, com menor risco de demissão, está sujeito a outros determinantes que caracterizam a precarização do seu trabalho, como a privatização das empresas públicas, a terceirização de setores, a degradação das suas condições de trabalho, a responsabilização pelas deficiências dos serviços, a instabilidade devida a mudanças políticas, as ações descontinuadas, o acúmulo de funções, além do estereótipo da morosidade, aspectos que podem afetar a saúde do trabalhador (NUNES; LINS, 2009).
Estudos sobre o perfil epidemiológico dos trabalhadores do serviço público ainda são escassos, especialmente a respeito das causas do absenteísmo. Pesquisas realizadas sobre o tema mostraram poucos estudos sobre as causas dos afastamentos do trabalho; muitas ainda têm como foco apenas os trabalhadores das áreas da saúde ou da educação. No entanto, os TMC aparecem recorrentemente como um fator que contribui de forma significativa para o aumento dos afastamentos do trabalho (FALAVIGNA, 2010; GEHRING JUNIOR et al., 2007; JACQUES; AMAZARRAY, 2006; NUNES; LINS, 2009).

No âmbito do serviço público federal, a criação de uma Política de Atenção à Saúde do Servidor entrou na pauta de debates desde 2005, o que culminou com a instituição do Subsistema Integrado de Atenção à Saúde do Servidor Público Federal (SIASS) através do Decreto n. 6.833, de 29 de abril de 2009 (BRASIL, 2009). Sua função principal é operacionalizar a Política de Atenção à Saúde e Segurança do Trabalho do Servidor Público Federal (PASS) através da coordenação de ações e programas nas áreas de assistência à saúde, perícia oficial, promoção, prevenção, vigilância e acompanhamento da saúde dos servidores da administração federal direta, autárquica e fundacional (BRASIL, 2009). As Unidades SIASS utilizam o Sistema Integrado de Administração de Recursos Humanos no módulo Saúde (SIAPE-Saúde) ${ }^{2}$ para registro eletrônico das informações epidemiológicas dos servidores e alimentam um banco de dados nacional.

Na prática profissional acredita-se, de forma empírica, que há uma grande quantidade de afastamentos, pedidos de remoção, de redistribuição e de readaptação motivados por TMC evidenciados não apenas nos atestados e laudos médicos, mas também no momento de realização das perícias e avaliações psicossociais. Diante disso, vislumbrou-se uma oportunidade de aliar os fatos dessa realidade com o interesse em realizar uma pesquisa na área da saúde mental e trabalho. Esta pesquisa também se tornou oportuna devido à construção de um banco de dados inédito sobre a saúde dos servidores federais, através do sistema SIAPE-Saúde, e à escassez de estudos que abarcassem as características epidemiológicas dos trabalhadores no setor público federal no Brasil, especialmente no estado do Tocantins.

Sendo assim, a presente pesquisa foi delineada com o objetivo de avaliar as características epidemiológicas dos afastamentos por TMC entre servidores públicos

\footnotetext{
2 O SIAPE-Saúde é um sistema informatizado da Administração Pública Federal gerido pela Secretaria de Recursos Humanos do Ministério do Planejamento, Orçamento e Gestão que consolida informações sobre a saúde dos servidores da administração pública de forma descentralizada e estruturada por meio dos módulos: Perícia Oficial, Exames Periódicos de Saúde, Promoção e Vigilância, Investidura em Cargo Público e Informações Gerenciais. O SIAPE-Saúde possibilita a gestão informatizada das informações sobre a saúde dos servidores pelas Unidades do Subsistema Integrado de Atenção à Saúde do Servidor (Unidades SIASS) (BRASIL, 2010).
} 
federais no Tocantins, no período de abril de 2011 a dezembro de 2012.

Por características epidemiológicas entende-se os atributos que identificam o agravo, a sua distribuição em relação às características da pessoa, tempo e lugar, fatores associados à sua ocorrência (determinantes) e extensão dos seus efeitos, entre outros aspectos. Sendo assim, para compreender o fenômeno dos afastamentos por transtornos mentais numa população formada por servidores públicos federais foi imprescindível conhecer a frequência de afastamentos do trabalho, a quantidade de dias de afastamento, a ocorrência de reincidência de afastamento por transtornos mentais e o tipo de perícia realizada.

\section{Métodos}

Esta é uma pesquisa exploratória de caráter transversal realizada a partir de dados armazenados no sistema SIAPE-Saúde sobre os afastamentos por motivo de saúde dos servidores públicos federais atendidos na Unidade SIASS/UFT/Palmas-TO.

O período da pesquisa compreende a data de início do funcionamento da Unidade SIASS/UFT/Palmas-TO, 4 de abril de 2011, até o último dia do ano de 2012, essa última em razão do limite estabelecido para o início da coleta dos dados, perfazendo dessa forma um intervalo de tempo de 20 meses e 27 dias (aproximadamente 21 meses) para a pesquisa.

O estudo contempla apenas servidores públicos federais ativos de órgãos do Poder Executivo no Tocantins, lotados em todos os municípios desse estado, que utilizaram os serviços da Unidade SIASS/UFT/Palmas-TO. Os órgãos dos Poderes Legislativo e Judiciário não são atendidos pela Unidade SIASS por não possuírem seus registros de afastamento contabilizados no sistema SIAPE-Saúde.

Os sujeitos da pesquisa foram selecionados utilizando-se os seguintes critérios de inclusão: ser servidor público federal ativo, ocupante de cargo em órgão do Poder Executivo no estado do Tocantins e ter apresentado histórico de afastamento por TMC no período de 4 de abril de 2011 a 31 de dezembro de 2012. Foram excluídos da pesquisa os sujeitos vinculados aos órgãos que não têm informações no sistema SIAPE-Saúde em razão de sigilo administrativo e aqueles que não possuíam registro de afastamento por diagnóstico de transtorno mental no intervalo de tempo pesquisado. Atendendo aos critérios supracitados, a população da pesquisa foi composta por 103 servidores distribuídos entre 12 dos 24 órgãos federais do Poder Executivo com sede no estado do Tocantins.

O instrumento de coleta de dados se constituiu em um banco de dados construído pela pesquisadora de acordo com as variáveis de interesse, contendo as informações dos prontuários eletrônicos selecionados a partir do sistema SIAPE-Saúde e as informações das pesquisas realizadas no sítio do Portal da Transparência do Governo Federal. As variáveis foram distribuídas em três blocos (sociodemográfico, ocupacional e epidemiológico) e detalhadas em um livro-código para facilitar o processo de organização dos dados durante a tabulação.

A pesquisa foi iniciada com a busca no sistema SIAPE-Saúde, através da qual foram coletados os dados da quantidade de afastamentos por TMC (que corresponde ao CID F - grupo F da Classificação Internacional de Doenças, $10^{\mathrm{a}}$ versão, CID-10) para cada órgão atendido pela Unidade SIASS/UFT/Palmas-TO no período do estudo. Na segunda etapa foram selecionados os órgãos com registro de afastamento por CID F e prosseguiu-se o levantamento dos prontuários eletrônicos de todos os servidores desses órgãos, de forma que fosse possível quantificar individualmente os registros de afastamento por CID F. A filtragem resultou no quantitativo absoluto de 103 prontuários eletrônicos que continham pelo menos um registro de afastamento por TMC no período estabelecido para a pesquisa.

As informações dos servidores colhidas no estudo dos prontuários foram trabalhadas de forma a preservar seu sigilo e nenhum servidor foi identificado pelo nome no processo de tabulação. Em seguida essas informações foram digitadas no banco de dados e codificadas numericamente. Através desses prontuários obteve-se o histórico individual dos afastamentos, assim como as informações para algumas das variáveis estabelecidas. Os dados ocupacionais (tempo no serviço público, tempo no órgão, remuneração bruta, cargo e carga horária) foram obtidos no Portal da Transparência do Governo Federal através da consulta livre na página sobre os Servidores Civis e Militares do Poder Executivo Federal. Dessas variáveis, apenas as três primeiras foram consideradas "fatores ocupacionais". Tal restrição foi necessária para atender às observações éticas e descartar a possibilidade de identificação dos servidores através de outras características como, por exemplo, qual cargo ocupa. A variável "Carga horária” também foi eliminada por não apresentar variação nos dados.

Para categorização e análise estatística, os dados foram tabulados em microcomputador no programa Statistical Package for the Social Sciences (SPSS) versão 20.0 para Windows. Foram elencadas as seguintes variáveis:

a) Campo sociodemográfico: Idade e sexo;

b) Campo ocupacional: Tempo no serviço público, tempo no órgão e remuneração; 
c) Campo epidemiológico: Número de afastamentos em geral, número de afastamentos por diagnóstico de TMC (CID F), dias de afastamento, mais de um afastamento por TMC, CID-10 registrado e tipo de perícia.

As variáveis se limitaram àquelas disponibilizadas no sistema SIAPE-Saúde bem como àquelas divulgadas no sítio do Portal da Transparência do Governo Federal. As informações do referido Sistema não contemplam outras características referentes ao ambiente de trabalho. Os prontuários eletrônicos registram apenas os códigos dos diagnósticos, não havendo descrição da gravidade do transtorno, tampouco categorização em escala.

A inexistência de padronização dos exames admissionais (que inclui avaliação clínica, exames físicos e de saúde mental) impossibilitou o levantamento de dados sobre o quadro prévio da saúde do servidor, não sendo possível verificar se os transtornos mentais precediam seu ingresso no serviço público federal.

Para descrever os dados coletados foram calculadas a média e o desvio-padrão. Foram empregados o teste do Qui-quadrado, os testes não paramétricos de Mann-Whitney e Kruskal-Wallis e o teste de correlação parcial com controle da variável "Idade", adotando-se o nível de significância de 5\% ( $\mathrm{p}<0,05)$. O cálculo de correlação foi feito para cada uma das variáveis numéricas "Tempo no serviço público", "Tempo no órgão" e "Remuneração" em relação ao número de afastamentos por TMC e à quantidade de dias de afastamento. Buscou-se identificar a relação e a intensidade da relação entre as variáveis ocupacionais e epidemiológicas quando o efeito da variável "Idade" foi controlado. Para interpretação da magnitude foi considerada correlação positiva forte os valores entre 0,7 a 1 , moderada entre 0,3 a 0,7 e fraca entre 0 a 0,3 ; e correlação negativa forte valores entre $-0,7$ a -1 , moderada entre $-0,3$ a $-0,7$ e fraca entre 0 a -0,3 (BARBETTA, 2006). A hipótese principal é a de que quanto maior for o tempo de trabalho no serviço público ou no órgão, maior é o número de casos de afastamento por TMC e maior é o tempo que o servidor fica afastado do trabalho em decorrência do transtorno.

O trabalho foi aprovado pelo Comitê de Ética em Pesquisa da Universidade Federal do Tocantins (parecer n. 088/2012, de 24/8/2012).

\section{Resultados}

As características sociodemográficas, ocupacionais e epidemiológicas gerais da população estudada estão descritas na Tabela 1. O quantitativo de servidores apresentou proporções equilibradas entre mulheres
( $\mathrm{n}=53,51,5 \%)$ e homens ( $\mathrm{n}=50,48,5 \%)$. Observou-se que a ocorrência de transtornos mentais está presente em todas as faixas etárias categorizadas, demonstrando-se que esse tipo de evento pode acontecer com servidores de qualquer idade. Verificou-se que nessa população, dos 339 afastamentos por motivo de saúde em geral (incluindo todos os CIDs), 176 (51,91\%) foram determinados por diagnóstico de TMC.

No que diz respeito ao tipo de perícia realizada, os resultados mostraram que $66(64,1 \%)$ dos casos de TMC foram avaliados mediante Perícia Singular, ou seja, realizada com pelo menos um médico ou um odontólogo; 20 (19,4\%) foram avaliados pela Junta Oficial, composta por três médicos ou três odontólogos; e 17 não passaram por qualquer tipo de perícia, uma vez que houve apenas o registro eletrônico dos afastamentos no sistema SIAPE-Saúde. Em geral, não necessitam passar por avaliação os casos que requerem um período de licença inferior a cinco dias consecutivos.

A distribuição dos subgrupos de CID F por sexo está descrita na Tabela 2. É necessário considerar que 31 servidores $(30,1 \%)$ da população estudada tiveram mais de um afastamento por TMC. Desses, 23 apresentaram co-ocorrência de dois ou mais desses transtornos, fenômeno que se denomina comorbidade.

Nessa tabela foi considerada a frequência dos registros de TMC por ocorrência de afastamento $(n=176)$, independentemente de estarem relacionados a um mesmo servidor, e categorizados em subgrupos de acordo com a CID-10. Dessa forma, a distribuição dos CIDs apresenta uma relação mais fidedigna com o número absoluto de afastamentos por TMC da população estudada.

Assim, os dados revelaram os subgrupos "Transtornos do humor (afetivos)" (CID F30 a F39) e "Transtornos neuróticos, transtornos relacionados com o stress e transtornos somatoformes" (CID F40 a F48) como os que apresentam maior número de afastamentos. No primeiro subgrupo, os "Episódios depressivos" (CID F32) são os mais representativos; no segundo subgrupo destacam-se as "Reações ao stress grave e transtornos de adaptação" (CID F43), seguidas de "Outros transtornos ansiosos" (CID F41).

A Tabela 3 mostra a comparação entre os grupos de variáveis sociodemográficas e ocupacionais, e as variáveis epidemiológicas em relação ao número absoluto de afastamentos e à quantidade de dias de afastamento. Para facilitar a tabulação dos dados foi contabilizado apenas um CID para cada servidor, utilizando-se como critério de seleção aquele que gerou a mais dias de afastamento e, portanto, maior absenteísmo ao trabalho. Esses foram denominados "CIDs prevalentes". O propósito foi avaliar os subgrupos do CID-F que se destacaram quando os 
Tabela 1 Características sociodemográficas, ocupacionais e epidemiológicas de servidores públicos federais com registro de afastamento por transtornos mentais e comportamentais (CID $\mathrm{F}^{*}$ ) no período de 4/4/11 a 31/12/12, no estado do Tocantins, Brasil

\begin{tabular}{|c|c|c|c|}
\hline Variáveis & $n(\%)$ & Média & Desvio-padrão \\
\hline \multicolumn{4}{|l|}{ Idade (anos) } \\
\hline Até 20 & - & & \\
\hline 21 a 30 & $14(13,6 \%)$ & & \\
\hline 31 a 40 & $38(36,9 \%)$ & & \\
\hline 41 a 50 & $25(24,2 \%)$ & & \\
\hline 51 a 60 & $22(21,4 \%)$ & & \\
\hline Mais de 60 & $4(3,9 \%)$ & & \\
\hline Total & $103(100 \%)$ & 42,03 & 10,35 \\
\hline \multicolumn{4}{|l|}{ Sexo } \\
\hline Mulher & $53(51,5 \%)$ & - & - \\
\hline Homem & $50(48,5 \%)$ & - & - \\
\hline \multicolumn{4}{|l|}{ Tempo no serviço público (anos) } \\
\hline Até 3 & $25(24,2 \%)$ & & \\
\hline 4 a 6 & $32(31,1 \%)$ & & \\
\hline 7 a 9 & $11(10,7 \%)$ & & \\
\hline 10 a 12 & - & & \\
\hline 13 a 15 & - & & \\
\hline 16 ou mais & $35(34 \%)$ & & \\
\hline Total & $103(100 \%)$ & 11,59 & 10,82 \\
\hline \multicolumn{4}{|l|}{ Tempo no órgão (anos) } \\
\hline Até 3 & $34(33 \%)$ & & \\
\hline 4 a 6 & $34(33 \%)$ & & \\
\hline 7 a 9 & $11(10,7 \%)$ & & \\
\hline 10 a 12 & $1(1 \%)$ & & \\
\hline 13 a 15 & - & & \\
\hline 16 ou mais & $23(22,3 \%)$ & & \\
\hline Total & $103(100 \%)$ & 8,18 & 8,25 \\
\hline \multicolumn{4}{|l|}{ Remuneração bruta (R\$) } \\
\hline Até 2.000,00 & $3(2,9 \%)$ & & \\
\hline $2.000,01$ a $4.000,00$ & $29(28,2 \%)$ & & \\
\hline $4.000,01$ a $6.000,00$ & $32(31,1 \%)$ & & \\
\hline $6.000,01$ a $8.000,00$ & $20(19,4 \%)$ & & \\
\hline $8.000,01$ a $10.000,00$ & $9(8,7 \%)$ & & \\
\hline Mais de $10.000,00$ & $10(9,7 \%)$ & & \\
\hline Total & $103(100 \%)$ & $5.953,39$ & $3.715,23$ \\
\hline Total de afastamentos para todos os CIDs & $339(100 \%)$ & 3,29 & 2,34 \\
\hline Afastamentos por CID F & $176(100 \%)$ & 1,71 & 1,23 \\
\hline \multicolumn{4}{|l|}{ Mais de um afastamento por CID F } \\
\hline Sim & $31(30,1 \%)$ & - & - \\
\hline Não & $72(69,9 \%)$ & - & - \\
\hline
\end{tabular}

"CID F: Grupo F da Classificação Internacional de Doenças, $10^{a}$ versão, que diz respeito aos diagnósticos de transtornos mentais e comportamentais. Fonte: Sistema SIAPE-Saúde (BRASIL, 2013d) e Portal da Transparência do Governo Federal (BRASIL, 2013e).

Continua.... 
Tabela 1 Continuação...

\begin{tabular}{|c|c|c|c|}
\hline Variáveis & $n(\%)$ & Média & Desvio-padrão \\
\hline \multicolumn{4}{|l|}{ Dias de afastamento por CID F } \\
\hline Até 15 dias & $46(44,6 \%)$ & & \\
\hline 16 a 30 dias & $17(16,5 \%)$ & & \\
\hline 31 a 45 dias & $8(7,8 \%)$ & & \\
\hline 46 a 60 dias & $7(6,8 \%)$ & & \\
\hline 61 a 75 dias & $4(3,9 \%)$ & & \\
\hline 76 a 90 dias & $3(2,9 \%)$ & & \\
\hline 91 a 105 dias & $4(3,9 \%)$ & & \\
\hline 106 a 120 dias & $4(3,9 \%)$ & & \\
\hline 121 dias ou mais & $10(9,7 \%)$ & & \\
\hline Total & $103(100 \%)$ & 43,92 & 50,91 \\
\hline \multicolumn{4}{|l|}{ Tipo de perícia } \\
\hline Singular & $66(64,1 \%)$ & - & - \\
\hline Junta & $20(19,4 \%)$ & - & - \\
\hline Não se aplica & $17(16,5 \%)$ & - & - \\
\hline
\end{tabular}

*CID F: Grupo F da Classificação Internacional de Doenças, $10^{\mathrm{a}}$ versão, que diz respeito aos diagnósticos de transtornos mentais e comportamentais. Fonte: Sistema SIAPE-Saúde (BRASIL, 2013d) e Portal da Transparência do Governo Federal (BRASIL, 2013e).

Tabela 2 Transtornos mentais e comportamentais (CID F*) segundo sexo de servidores públicos federais com registro de afastamento no período de 4/4/11 a 31/12/12, no estado do Tocantins, Brasil

\begin{tabular}{|c|c|c|c|}
\hline \multirow[b]{2}{*}{ CID F* por subgrupo } & \multicolumn{3}{|c|}{ Sexo (frequência) } \\
\hline & $\begin{array}{c}\text { Mulher } \\
n(\%) * * * 6\end{array}$ & $\begin{array}{l}\text { Homem } \\
n(\%) * * * * 6\end{array}$ & $\begin{array}{l}\text { Total } \\
n(\%)\end{array}$ \\
\hline F00 a F09 - Transtornos mentais orgânicos & $2(1,1 \%)$ & - & $2(1,1 \%)$ \\
\hline F10 a F19 - TMC devido ao uso de substância psicoativa & $5(2,8 \%)$ & $8(4,6 \%)$ & $13(7,4 \%)$ \\
\hline F20 a F29 - Esquizofrenia, transtornos esquizotípicos e transtornos delirantes & $6(3,4 \%)$ & $4(2,4 \%)$ & $10(5,8 \%)$ \\
\hline F30 a F39 - Transtornos do humor (afetivos) & $35(19,9 \%)$ & $30(17,0 \%)$ & $65(36,9 \%)$ \\
\hline F32 - Episódios depressivos & $20(11,3 \%)$ & $21(11,9 \%)$ & $41(23,2 \%)$ \\
\hline F40 a F48 - Transtornos neuróticos, relacionados com stress e somatoformes & $46(26,1 \%)$ & $34(19,4 \%)$ & $80(45,5 \%)$ \\
\hline F41 - Outros transtornos ansiosos & $17(9,6 \%)$ & $17(9,6 \%)$ & $34(19,2 \%)$ \\
\hline F43 - Reações ao stress grave e transtornos de adaptação & $23(13 \%)$ & $15(8,5 \%)$ & $38(21,5 \%)$ \\
\hline $\begin{array}{l}\text { F50 a F59 - Síndromes comportamentais associadas a disfunções fisiológicas e a } \\
\text { fatores físicos }\end{array}$ & - & $1(0,5 \%)$ & $1(0,5 \%)$ \\
\hline F60 a F69 - Transtorno da personalidade e do comportamento do adulto & $1(0,5 \%)$ & - & $1(0,5 \%)$ \\
\hline F70 a F79 - Retardo mental & - & - & - \\
\hline F80 a F89 - Transtornos do desenvolvimento psicológico & - & - & - \\
\hline $\begin{array}{l}\text { F90 a F98 - Transtornos do comportamento e emocionais que aparecem habitual- } \\
\text { mente durante a infância ou adolescência }\end{array}$ & $4(2,3 \%)$ & - & $4(2,3 \%)$ \\
\hline F99-Transtorno mental não especificado & - & - & - \\
\hline Total & $99(56,2 \%)$ & $77(43,8 \%)$ & $176(100 \%)$ \\
\hline
\end{tabular}

*CID F: Grupo F da Classificação Internacional de Doenças, $10^{a}$ versão, que diz respeito aos diagnósticos de transtornos mentais e comportamentais.

*****ercentagem em relação ao total (176).

Fonte: Sistema SIAPE-Saúde (BRASIL, 2013d). 
dados foram cruzados com o número de afastamentos e a quantidade de dias de afastamento. Ou seja, ao considerarmos apenas um diagnóstico de TMC para cada servidor, os “Transtornos do humor (afetivos)” e os "Transtornos neuróticos, transtornos relacionados com o stress e transtornos somatoformes" são os mais representativos.

$\mathrm{Na}$ comparação entre os grupos, as variáveis "Idade”, "Sexo”, “Tempo no serviço público”, “Tempo no órgão" e "Remuneração", quando comparadas com

Tabela 3 Comparação entre variáveis sociodemográficas, ocupacionais e epidemiológicas e o número de afastamentos e dias de afastamento para tratamento de saúde por transtornos mentais e comportamentais (CID $\mathrm{F}^{*}$ ) entre servidores públicos federais com registro de afastamento no período de 4/4/11 a $31 / 12 / 12$ no estado do Tocantins, Brasil $(\mathrm{n}=103)$

\begin{tabular}{|c|c|c|c|c|c|c|c|c|c|}
\hline \multirow[b]{2}{*}{ Variáveis } & \multicolumn{5}{|c|}{ Número de afastamentos } & \multicolumn{4}{|c|}{ Dias de afastamento } \\
\hline & $n^{*}$ & Média & $\begin{array}{l}\text { Desvio- } \\
\text { padrão }\end{array}$ & $p^{* * *}$ & $Z^{* * * * * *}$ & Média & $\begin{array}{l}\text { Desvio- } \\
\text { padrão }\end{array}$ & $p^{* * *}$ & $Z^{* * * * * *}$ \\
\hline \multicolumn{10}{|l|}{ Idade } \\
\hline Até 20 & - & - & - & \multirow{6}{*}{0,86} & \multirow{6}{*}{1,30} & - & - & \multirow{6}{*}{0,33} & \multirow{6}{*}{4,63} \\
\hline 21 a 30 & 14 & 1,79 & 1,63 & & & 29,00 & 38,35 & & \\
\hline 31 a 40 & 38 & 1,71 & 1.14 & & & 47,55 & 56,33 & & \\
\hline 41 a 50 & 25 & 1,84 & 1,43 & & & 55,88 & 57,16 & & \\
\hline 51 a 60 & 22 & 1,45 & 0,80 & & & 32,59 & 41,33 & & \\
\hline Mais de 60 & 4 & 2,00 & 1,41 & & & 49,25 & 33,49 & & \\
\hline \multicolumn{10}{|l|}{ Sexo } \\
\hline Mulher & 53 & 1,89 & 1,44 & \multirow{2}{*}{0,19} & \multirow{2}{*}{$1.493,50$} & 44,34 & 54,73 & \multirow{2}{*}{0,72} & \multirow{2}{*}{$1.271,00$} \\
\hline Homem & 50 & 1,52 & 0,93 & & & 43,48 & 47,06 & & \\
\hline \multicolumn{10}{|l|}{ Tempo no serviço público } \\
\hline Até 3 & 25 & 2,12 & 1,67 & \multirow{6}{*}{0,14} & \multirow{6}{*}{5,39} & 61,40 & 65,46 & \multirow{6}{*}{0,30} & \multirow{6}{*}{3,66} \\
\hline 4 a 6 & 32 & 1,66 & 1,21 & & & 37,81 & 52,57 & & \\
\hline 7 a 9 & 11 & 1,73 & 0,90 & & & 61,18 & 53,95 & & \\
\hline 10 a 12 & - & - & - & & & - & - & & \\
\hline 13 a 15 & - & - & - & & & - & - & & \\
\hline 16 ou mais & 35 & 1,46 & 0,89 & & & 31,60 & 29,33 & & \\
\hline \multicolumn{10}{|l|}{ Tempo no órgão } \\
\hline Até 3 & 31 & 2,03 & 1,55 & \multirow{6}{*}{0,26} & \multirow{6}{*}{5,26} & 58,56 & 63,28 & \multirow{6}{*}{0,07} & \multirow{6}{*}{8,70} \\
\hline 4 a 6 & 34 & 1,59 & 1,16 & & & 34,06 & 45,38 & & \\
\hline 7 a 9 & 11 & 1,73 & 0,90 & & & 70,18 & 49,09 & & \\
\hline 10 a 12 & 1 & 1,00 & - & & & 30,00 & - & & \\
\hline 13 a 15 & - & - & - & & & - & - & & \\
\hline 16 ou mais & 23 & 1,43 & 0,84 & & & 24,91 & 24,70 & & \\
\hline \multicolumn{10}{|l|}{ Remuneração } \\
\hline Até $2.000,00$ & 3 & 1,67 & 0,58 & \multirow{6}{*}{0,89} & \multirow{6}{*}{1,69} & 53,67 & 83,50 & \multirow{6}{*}{0,74} & \multirow{6}{*}{2,71} \\
\hline $2.000,01$ a $4.000,00$ & 29 & 2,00 & 1,58 & & & 40,34 & 50,32 & & \\
\hline $4.000,01$ a $6.000,00$ & 32 & 1,75 & 1,32 & & & 54,25 & 56,58 & & \\
\hline $6.000,01$ a $8.000,00$ & 20 & 1,45 & 0,83 & & & 35,95 & 46,93 & & \\
\hline $8.000,01$ a $10.000,00$ & 9 & 1,44 & 0,73 & & & 26,89 & 27,05 & & \\
\hline Mais de $10.000,00$ & 10 & 1,50 & 0,85 & & & 49,60 & 51,93 & & \\
\hline
\end{tabular}

*Número absoluto

Nível de significância estabelecido $\mathrm{p}<0,05$.

***:* Estatística de teste de hipótese; Para comparação de dois grupos independentes utilizou-se o teste não paramétrico de Mann-Whitney; Para três ou mais grupos independentes utilizou-se o teste não paramétrico Kruskal-Wallis.

Continua.... 
Tabela 3 Continuação...

\begin{tabular}{|c|c|c|c|c|c|c|c|c|c|}
\hline \multirow[b]{2}{*}{ Variáveis } & \multicolumn{5}{|c|}{ Número de afastamentos } & \multicolumn{4}{|c|}{ Dias de afastamento } \\
\hline & $n^{*}$ & Média & $\begin{array}{l}\text { Desvio- } \\
\text { padrão }\end{array}$ & $p^{\text {*** }}$ & $Z^{* * * * *}$ & Média & $\begin{array}{l}\text { Desvio- } \\
\text { padrão }\end{array}$ & $p^{* * *}$ & $Z^{* * * * *}$ \\
\hline \multicolumn{10}{|l|}{ CID (prevalente) } \\
\hline F00 a F09 & 2 & 1,00 & - & & & 16,00 & 19,80 & & \\
\hline F10 a F19 & 6 & 2,00 & 1,26 & & & 70,83 & 51,59 & & \\
\hline F20 a F29 & 5 & 2,20 & 1,64 & & & 65,40 & 87,35 & & \\
\hline F30 a F39 & 43 & 1,63 & 0,98 & & & 45,07 & 48,54 & & \\
\hline $\mathrm{F} 40$ a F48 & 43 & 1,74 & 1,45 & & & 41,40 & 50,40 & & \\
\hline F50 a F59 & 1 & 1,00 & - & 0,40 & 19,97 & 07,00 & - & 0,04 & 11,19 \\
\hline F60 a F69 & 1 & 1,00 & - & & & 06,00 & - & & \\
\hline F70 a F79 & - & - & - & & & - & - & & \\
\hline F80 a F89 & - & - & - & & & - & - & & \\
\hline F90 a F98 & 2 & 2,00 & 1,41 & & & 04,50 & 4,95 & & \\
\hline F99 & - & - & - & & & - & - & & \\
\hline \multicolumn{10}{|l|}{ Tipo de perícia } \\
\hline Singular & 66 & 1,39 & 0,80 & & & 32,42 & 31,15 & & \\
\hline Junta & 20 & 2,90 & 1,83 & 0,00 & 20,45 & 115,9 & 56,87 & 0,00 & 60,12 \\
\hline Não se aplica & 17 & 1,53 & 0,87 & & & 3,88 & 3,238 & & \\
\hline
\end{tabular}

*Número absoluto.

栐Nível de significância estabelecido $\mathrm{p}<0,05$.

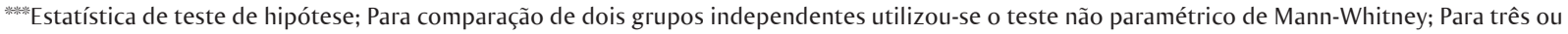
mais grupos independentes utilizou-se o teste não paramétrico Kruskal-Wallis.

o "Número de afastamentos" e "Quantidade de dias de afastamento", apresentaram um p-valor maior que o estipulado para o teste estatístico. Dessa forma pôde-se verificar que não se encontram diferenças estatísticas significativas para essas variáveis em estudo. Todavia as variáveis "CID (prevalente)" e "Tipo de perícia”, quando submetidas à mesma comparação, apresentaram significância estatística $(p<0,05)$ na diferença das médias.

Na variável "CID (prevalente)", as doenças que, em média, mais afastaram foram, nessa ordem, "Esquizofrenia, transtornos esquizotípicos e transtornos delirantes" ( $\mathrm{M}=2,20$ afastamentos/servidor; $\mathrm{DP}=1,64)$; "Transtornos do comportamento e emocionais que aparecem habitualmente durante a infância ou adolescência" ( $\mathrm{M}=2,00$ afastamentos/servidor; $\mathrm{DP}=1,41$ ), "Transtornos neuróticos, relacionados com o stress e somatoformes" ( $\mathrm{M}=1,74 ; \mathrm{DP}=1,45)$ e "Transtornos do humor (afetivos)" ( $\mathrm{M}=1,63$; $\mathrm{DP}=0,98)$. Aquelas que, em média, geraram maior quantidade de dias de afastamento foram, respectivamente, "Transtornos mentais devido ao uso de substâncias psicoativas" ( $\mathrm{M}=70,83$ dias de afastamento/servidor; $\mathrm{DP}=51,59$ ), "Esquizofrenia, transtornos esquizotípicos e transtornos delirantes" ( $\mathrm{M}=65,40$; $\mathrm{DP}=87,35)$ e "Transtornos do humor (afetivos)" ( $\mathrm{M}=45,07$; $\mathrm{DP}=48,54)$. Quanto ao tipo de perícia, a Junta Oficial apresentou as maiores médias para as duas variáveis epidemiológicas de comparação, justamente por ser essa a modalidade de avaliação pericial que julga os casos mais complexos de TMC, os quais, em geral, afastam o servidor com mais frequência e por períodos que ultrapassam 120 dias por ano.

A análise da correlação parcial revelou que, ao eliminar o efeito da idade, as variáveis "Tempo no serviço público" e "Tempo no órgão", quando correlacionadas ao número de afastamentos por TMC, não apresentaram resultados de significância estatística $(\mathrm{r}=-0,176 / \mathrm{p}=0,077$ e $\mathrm{r}=-0,143 / \mathrm{p}=0,152$, respectivamente). O mesmo ocorreu com as correlações da variável "Remuneração" em relação ao número de afastamentos por TMC ( $\mathrm{r}=-0,093 / \mathrm{p}=0,352)$ e à quantidade de dias de afastamento $(\mathrm{r}=-0,022 / \mathrm{p}=0,823)$.

Todavia, o coeficiente de correlação das variáveis "Tempo no serviço público" e "Tempo no órgão" apresenta uma correlação negativa fraca em relação à quantidade de dias de afastamento $(\mathrm{r}=-0,254 / \mathrm{p}=0,010$ e $\mathrm{r}=-0,220 / \mathrm{p}=0,026$, respectivamente), indicando que na população em estudo os servidores com menos tempo de trabalho no serviço público apresentaram quantidades maiores de dias de afastamento em razão da ocorrência de TMC.

A Figura 1 apresenta os gráficos de dispersão da correlação entre as variáveis "Tempo no serviço público" e "Tempo no órgão" em relação aos "Dias de afastamento", e suas respectivas linhas de tendência. 

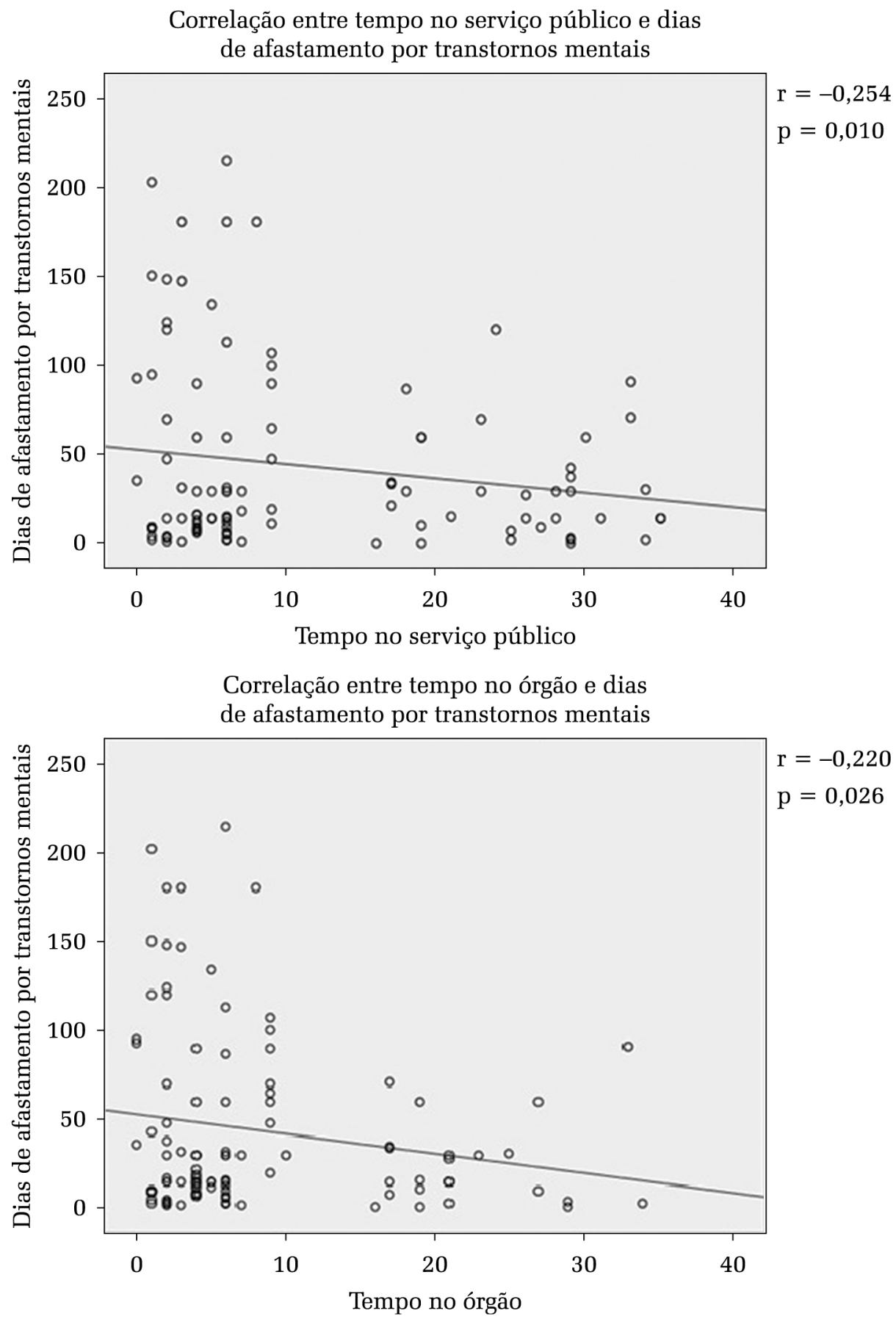

Figura 1 Correlação entre as variáveis “Tempo no serviço público" e "Tempo no órgão" em relação aos "Dias de afastamento" de servidores públicos federais com registro de afastamento por transtornos mentais e comportamentais (CID F*) no período de 4/4/11 a 31/12/12, no estado do Tocantins, Brasil

\section{Discussão}

Segundo o Boletim Estatístico de Pessoal publicado pelo Ministério do Planejamento, Orçamento e Gestão em novembro de 2013, o Brasil tem 541.497 servidores públicos ativos vinculados ao Poder Executivo, dos quais $4.061(0,75 \%)$ estão lotados no estado do Tocantins, um quantitativo que é representado por 2.447 homens e 1.614 mulheres. A idade média desses servidores é de 41 anos, cinco anos a menos que a média nacional (46 anos) (BRASIL, 2013c). 
Os afastamentos por TMC identificados na pesquisa ( $\mathrm{n}=176$ ) totalizam 4.524 dias não trabalhados ${ }^{3}$, numa média de aproximadamente 44 dias de afastamento por servidor. Demyttenaere et al. (2004) afirmam que os transtornos mentais estão entre as principais causas de perda de dias de trabalho. Os casos leves causam perda de quatro dias de trabalho/ano e os graves, de cerca de 200 dias de trabalho/ano, em média. Esses quadros são frequentes e comumente incapacitantes, evoluindo para o absenteísmo e a redução da produtividade (NIEUWENHUIJSEN et al., 2006). De acordo com Whittaker et al. (2012), que conduziu um estudo sobre a relação entre transtornos mentais e absenteísmo em diferentes ocupações no Reino Unido, os maiores índices de afastamento do trabalho estavam entre servidores públicos.

Em um cálculo ilustrativo, dividindo a média salarial dos servidores pesquisados ( $\mathrm{R} \$ 5.953,39)$ por 30 dias (mês), obtém-se o valor de R\$198,44, que representa a estimativa média de quanto vale um dia de trabalho de cada indivíduo dessa população. Dessa forma, apenas no intervalo de 21 meses da pesquisa, os dias não trabalhados geraram para o Estado um custo estimado em R\$ 897.742,56.

Análises preliminares da Secretaria de Recursos Humanos do Ministério do Planejamento realizadas em 2006, com base em estudos da Organização Mundial da Saúde (OMS), indicavam que para cada $\mathrm{R} \$ 1,00$ investido em prevenção de doenças e de agravos ocupacionais, R $\$ 16,00$ são economizados em ações reparadoras. Esses investimentos, em tese, diminuem o número de afastamentos, o absenteísmo ou as aposentadorias por invalidez (BRASIL, 2006). Esses dados reforçam a necessidade de investimentos em ações eficazes de prevenção de agravos e de promoção à saúde do trabalhador no serviço público. Contudo, tais esforços devem acontecer não apenas no sentido de otimização dos recursos e redução de gastos, mas de promoção de uma melhor qualidade de vida no trabalho no âmbito do serviço público federal.

Para Morrone et al. (2004), o processo de adoecimento incapacitante entre servidores públicos também pode ser explicado pela falta de flexibilidade organizacional no sistema público. Há dificuldades para a implementação de ações de promoção de saúde e segurança do trabalho voltadas para o seu quadro de funcionários (SILVA JÚNIOR, 2012).

Dados compilados do SIAPE-Saúde demonstram que, no período delimitado nesta pesquisa, as três principais causas de afastamento entre os servidores federais no Tocantins foram: em primeiro lugar, as doenças do sistema osteomuscular e do tecido conjuntivo, seguidas das lesões, envenenamento e algumas outras consequências de causas externas e os transtornos mentais e comportamentais. Por consequência, esses também são os diagnósticos que possuem maior quantidade de dias de afastamento.

Observou-se um número significativo de afastamentos por "Episódios depressivos, reações ao stress grave e transtornos de adaptação"; e "Outros transtornos ansiosos". Andrade, Viana e Silveira (2006) realizaram um estudo similar a respeito da prevalência de absenteísmo entre trabalhadores de um serviço público municipal e verificaram que $81,6 \%$ dos 301 sujeitos pesquisados com transtornos mentais e comportamentais apresentavam quadros depressivo-ansiosos. Esses transtornos, segundo a literatura, também apresentam relação com o ambiente de trabalho (SANDERSON; ANDREWS, 2006; MENTAL..., 2010; CAVALHEIRO; TOLFO, 2011; VEMER et al., 2013; ARENDS et al., 2010). Um estudo longitudinal realizado sobre a relação entre os fatores organizacionais e sintomas depressivos entre enfermeiras concluiu que há um efeito direto entre os fatores da organização do trabalho e a saúde dos trabalhadores (LAMY et al., 2013).

Esses três principais diagnósticos de TMC também foram evidenciados em outro estudo realizado por Cavalheiro e Tolfo (2011) junto a servidores atendidos pela Junta Médica Oficial de uma universidade pública brasileira, que chega à conclusão de que tais transtornos colaboram para a incapacidade dos sujeitos ao trabalho.

O Transtorno ansioso ou Transtorno de ansiedade social

[...] é o terceiro problema de saúde mental mais comum, depois da depressão e do abuso do álcool. Ele possui curso crônico associado a um considerável comprometimento funcional, podendo ser incapacitante em suas formas mais graves. [...] (MARTINEZ et al., 2012, p. 283)

Esse transtorno é caracterizado pelo Manual de Diagnóstico e Estatístico de Transtornos Mentais, $4^{a}$ edição (ASSOCIAÇÃO PSIQUIÁTRICA AMERICANA, 2003), como um medo acentuado de situações sociais ou de desempenho nas quais o indivíduo teme sentir-se envergonhado ou embaraçado. Em casos graves, pode existir a perda da autoestima e medos oriundos de possíveis críticas. Os Transtornos depressivos, por sua vez, caracterizam-se por humor triste, perda do interesse e do prazer nas atividades cotidianas, sendo comum uma sensação de fadiga aumentada (ORGANIZAÇÃO MUNDIAL DA SAÚDE, 1998; CAVALHEIRO; TOLFO, 2011).

\footnotetext{
3 Se, hipoteticamente, todos esses dias de afastamento se referissem a apenas 1 (um) servidor, numa linha de tempo contínua, esse afastamento representaria aproximadamente 12,4 anos não trabalhados.
} 
Segundo Trigo, Teng e Hallak (2007, p. 230),

[...] há muitas indicações de que situações estressantes de origens familiar e laboral são fatores de risco para o desenvolvimento de desordens relacionadas ao estresse.

É possível que os diagnósticos de TMC evidenciados nesta pesquisa estejam associados a diversos fatores estressores que fazem parte do cotidiano de trabalho dos servidores públicos, tais como: intensa demanda dos serviços, precarização das estruturas físicas e de gestão pública, mudanças políticas de governo que alteram as rotinas e processos de trabalho, entre outros. Todavia, é necessário considerar a particularidade do serviço público no estado do Tocantins ser constituído por pessoas advindas de diferentes localidades, além do sentimento de não pertencimento, problemas de adaptação ao clima e a ruptura, ainda que parcial, das relações sociais preexistentes nas localidades de origem do servidor; tais fatores podem influenciar a saúde mental dos sujeitos e agir como desencadeadores dos transtornos mentais, especialmente transtornos de ansiedade associados à separação e transtornos de adaptação.

Analisando-se a distribuição dos grupos dos transtornos mentais em relação ao sexo dos sujeitos pesquisados, observou-se um equilíbrio entre homens e mulheres nos casos de episódios depressivos. Embora esse resultado não aponte predominância de um único sexo em relação ao número de casos de depressão, algumas linhas de estudos defendem que há uma tendência de a ocorrência de depressão ser mais comum entre mulheres. Andrade, Viana e Silveira (2006) realizaram um amplo estudo epidemiológico dos transtornos psiquiátricos na mulher, em diferentes fases da vida, avaliando diversas publicações científicas na área, e chegaram à conclusão de que há diferenças de sexo na ocorrência de transtornos mentais.

Justo e Calil (2006), numa revisão de literatura sobre o acometimento de depressão entre homens e mulheres, fazem menção a outras pesquisas que sugerem que a depressão em mulheres está associada aos diversos papéis sociais que a mulher acumulou ao longo dos anos, que aumentaram suas responsabilidades e a sobrecarga de trabalho.

Andrade, Viana e Silveira (2006) destacam que a co-ocorrência de dois ou mais transtornos (comorbidade) é um fenômeno comum tanto em amostras clínicas como em estudos populacionais. Segundo os autores, nesses casos as mulheres apresentam maior comorbidade entre episódios depressivos e transtornos de ansiedade, enquanto os homens apresentam comorbidade entre problemas relacionados ao uso de substâncias psicoativas e transtornos de conduta (ANDRADE; VIANA; SILVEIRA, 2006, p. 45-46).
O Relatório da Organização Mundial da Saúde sobre Saúde Mental destaca que é comum a ocorrência simultânea de dois ou mais transtornos mentais no mesmo indivíduo. Afirma que a ansiedade e os distúrbios depressivos frequentemente ocorrem juntos e que se observa essa comorbidade em aproximadamente metade das pessoas que apresentam qualquer um desses transtornos (MARTINEZ et al., 2012).

Em relação ao trabalho, um estudo de caso-controle realizado com 385 pessoas sobre os fatores associados aos afastamentos do trabalho por transtornos mentais indicou, assim como nesta pesquisa, que "Episódios depressivos", "Outros transtornos ansiosos" e "Reações ao stress grave e transtornos de adaptação" foram, nessa ordem, os transtornos mais prevalentes. $\mathrm{O}$ autor identificou ainda que, em relação aos fatores ocupacionais, houve associação estatística entre a incapacidade laborativa de longa duração e o vínculo de trabalho com empresa de natureza pública (SILVA JÚNIOR, 2012, p. 74). Houve também associação entre os afastamentos prolongados por transtornos mentais e o relato de mais de duas morbidades de saúde no ano anterior (SILVA JÚNIOR, 2012, p. 78).

Em números absolutos, a nossa pesquisa sinalizou 35 afastamentos por transtornos mentais entre servidores com mais de 16 anos de trabalho no serviço público federal. É preciso considerar que servidores dessa parcela acompanharam nas suas vivências laborais as transformações do mundo do trabalho impressas nas relações, técnicas, tecnologias e culturas organizacionais adotadas nos mais diversos ambientes de trabalho no serviço público federal. Essas metamorfoses, ainda que a longo prazo, influenciaram e continuam influenciando o sentido do trabalho para esses sujeitos. Dessa forma, experiências e sentimentos acumulados de mal-estar ao longo dos anos no ambiente organizacional potencializam os riscos de adoecimento.

Estudos realizados para investigar a relação entre saúde mental e trabalho já apontaram como algumas particularidades intrínsecas no contexto de trabalho no serviço público influenciam o adoecimento. Segundo Pires e Macêdo (2006, p. 96)

[...] as organizações públicas mantêm as mesmas
características básicas das demais organizações,
acrescidas, entretanto, de algumas especificidades
como: apego às regras e rotinas, supervalorização
da hierarquia, paternalismo nas relações, apego ao
poder, entre outras.

Tais características incidem sobre a satisfação do servidor quanto ao seu trabalho e, consequentemente, sobre sua saúde, conforme evidenciaram Faragher et al. (2005) em uma meta-análise baseada em 485 estudos, a qual apontou uma forte correlação entre os níveis de satisfação no trabalho e a saúde mental dos 
trabalhadores. Para os autores há evidências de que as mudanças nas condições de trabalho ocasionadas, principalmente, pelos avanços tecnológicos, possam contribuir para a diminuição dos níveis de satisfação do trabalhador (FARAGHER et al., 2005).

Outra pesquisa conduzida em 2005 com cerca de 30 mil trabalhadores em países europeus revelou que o absenteísmo do trabalho por motivo de doença está diretamente associado à realização de um trabalho monótono, sem o aprendizado de novas habilidades e com pouco controle do trabalhador sobre o trabalho realizado, assim como pouca participação dele na tomada de decisões (PARENT-THIRION et al., 2007). Em outro estudo, Elovainio et al. (2013) sugerem, ainda, que a baixa justiça organizacional também é um fator de risco para o absenteísmo por doenças relacionadas, principalmente, aos transtornos de ansiedade.

Os resultados deste trabalho apontaram que para cada servidor com até três anos de vínculo laboral com o serviço público houve uma média de 2,12 afastamentos relacionados aos transtornos mentais e comportamentais no período delimitado na pesquisa, configurando a maior média de afastamentos/servidor da variável “Tempo no serviço público”. Essa mesma constatação ocorreu entre servidores com até três anos de vínculo no órgão de lotação atual, cuja média foi de 2,03 afastamentos por servidor. Servidores com tempo de trabalho inferior a três anos são considerados em fase de estágio probatório. As hipóteses explicativas para esse resultado são diversas, mas imprecisas, e podem envolver desde fatores decorrentes da mudança de estado, tendo em vista que a maioria dos servidores lotados no Tocantins vem de várias origens, até fatores relacionados à má adaptação desses novos servidores às rotinas e funções no serviço público. Anchieta et al. (2011), em um estudo sobre o trabalho e os riscos de adoecimento entre servidores públicos da Polícia Civil do Distrito Federal, apontaram que na percepção dos policiais a organização, as condições e as relações interpessoais são elementos críticos do cotidiano de trabalho que sinalizam a possibilidade de adoecimento, especialmente para os servidores que estão há pouco tempo no cargo. Os autores destacam que ao se depararem com situações de pouca organização e de más condições de trabalho, os profissionais em início de carreira têm suas expectativas frustradas, o que, a longo prazo, potencializa o risco de adoecimento caso não sejam criadas estratégias de mediação para promoção da saúde e prevenção de agravos (ANCHIETA et al., 2011).

Outra hipótese é que esses servidores em estágio probatório afastados do trabalho por diagnóstico de transtornos mentais já tenham ingressado adoecidos no serviço público. Todavia, apesar dessas pressuposições, ainda não é possível ter uma explicação assertiva, uma vez que até o momento não foram encontrados estudos que investigassem detalhadamente as causas dos transtornos mentais numa população específica formada por servidores públicos, embora a resposta a essa questão seja essencial para o aprimoramento das ações de promoção à saúde do servidor.

Apesar da existência de algumas limitações, esta pesquisa amplia as possibilidades de se explorar um tema de relevância teórica e social em um estado (Tocantins) que possui 9,43\% da população ocupada atuando na Administração Pública (INSTITUTO BRASILEIRO DE GEOGRAFIA E ESTATÍSTICA, 2010). Até o momento não foram encontradas outras pesquisas do gênero que abarquem os servidores públicos em sua diversidade de cargos, lotações, atribuições etc. no âmbito da esfera federal. Este estudo é pioneiro na investigação da ocorrência de TMC numa população formada por servidores efetivos do Poder Executivo Federal no estado do Tocantins e na utilização, como referência, de uma base de dados inédita sobre a saúde dos servidores federais (SIAPE-Saúde). Embora não tenha sido comprovada uma relação direta dos transtornos mentais com os fatores ocupacionais, a existência desse fenômeno no serviço público pode estar associada a outras variáveis intrínsecas ou extrínsecas ao ambiente de trabalho que ainda precisam ser investigadas.

Não deixa de ser intrigante os transtornos mentais representarem uma proporção de $29,2 \%$ de todos os afastamentos de servidores federais no Tocantins registrados no SIAPE-Saúde no período da pesquisa $(\mathrm{n}=602)$. Apesar de alarmantes, os números de casos de transtornos mentais apresentados neste estudo não representam a totalidade de casos existentes no serviço público federal do Tocantins; a grande maioria sequer chega a ser registrada, especialmente devido ao receio da exposição e à existência de uma cultura preconceituosa consolidada nos próprios ambientes de trabalho.

Os resultados obtidos nesta pesquisa não apenas reforçam o que a Organização Mundial da Saúde (2001) já previa como tendência no campo da saúde pública, que era o crescimento dos casos de transtornos mentais potencialmente incapacitantes na população em geral, mas alertam para a necessidade de se reforçarem as concepções de saúde mental e de trabalho como indissociáveis na conjuntura produtiva atual, as quais perpassam o ambiente do serviço público em suas diversas dimensões. Por esse motivo ressalta-se a necessidade de ampliar-se o olhar sobre o absenteísmo-doença no serviço público para além da urgência da redução dos custos financeiros, mas compreendendo também o custo humano gerado pelo adoecimento por transtornos mentais. Para isso deverão ser realizadas novas pesquisas no sentido de se investigar os determinantes desses afastamentos, para então inferir se suas causas estão relacionadas 
aos fatores ocupacionais. Pelo que já apontaram as pesquisas realizadas nessa área, assim como as estatísticas sobre a saúde mental entre trabalhadores ativos, torna-se imprescindível a continuidade dos investimentos em políticas eficientes de suporte em saúde mental. No serviço público federal, os dados sobre os afastamentos por transtornos mentais ainda são incipientes, mas já alertam para a urgência de uma macropolítica de promoção à saúde e prevenção de agravos que ultrapasse os limites da minimização de impactos e promova uma melhor qualidade de vida no trabalho.

\section{Contribuições de autoria}

Todos os autores contribuíram substancialmente no levantamento, na análise dos dados e no processo de elaboração e revisão do texto.

\section{Referências}

ANCHIETA, V. C. C. et al. Trabalho e riscos de adoecimento: um estudo entre policiais civis. Psicologia: Teoria e Pesquisa, Brasília, v. 27, n. 2, p. 199-208, 2011.

ANDRADE, L. H. S. G.; VIANA, M. C.; SILVEIRA, C. M. Epidemiologia dos transtornos psiquiátricos na mulher. Revista de Psiquiatría Clínica, Santiago, v. 33, n. 2, p. 43-54, 2006. http://dx.doi.org/10.1590/S010160832006000200003 .

ARENDS, I.; VAN DER KLINK, J. J. L.; BÜLTMANN, U. Prevention of recurrent sickness absence in workers with common mental disorders: results of a cluster-randomised controlled trial. Occupational and Environmental Medicine, London, v. 10, n. 132, p. 1-9, 2010. PMid:24158311.

ASSOCIAÇÃO PSIQUIÁTRICA AMERICANA APA. DSM-IV: manual diagnóstico e estatístico de transtornos mentais. Tradução Dayse Batista. Porto Alegre: ARTMED, 2003.

BARBETTA, P. A. Estatística aplicada às Ciências Sociais. 6. ed. Florianópolis: Editora da UFSC, 2006.

BRASIL. Ministério de Planejamento, Orçamento e Gestão. Investimento em saúde do servidor previne aposentadoria precoce. Contato: Boletim do Servidor, Brasília, n. 43, p. 2, 2005.

. Ministério do Planejamento. Orçamento e Gestão. Coordenação Geral de Planejamento, Orçamento e Finanças. Ministério do Planejamento, Orçamento e Gestão - MP: relatório de atividades 2005. Brasília, 2006. 94p.

. Decreto $\mathrm{n}^{\mathrm{O}}$ 6.833, de 29 de abril de 2009. Institui o Subsistema Integrado de Atenção à Saúde do Servidor Público Federal - SIASS e o Comitê Gestor de Atenção à Saúde do Servidor. Diário Oficial [da] República Federativa do Brasil, Brasília, DF, 30 abr. 2009.

. Ministério do Planejamento. Orçamento e Gestão. Secretaria de Recursos Humanos. Manual de perícia oficial em saúde do servidor público federal. Brasília, 2010. 339p.
. Ministério da Previdência Social. Acompanhamento mensal dos benefícios auxíliosdoença acidentários concedidos segundo os códigos da CID-10. Brasília, 2013a. Janeiro a agosto de 2013 [tabelas].

. Ministério da Previdência Social. Acompanhamento mensal dos benefícios auxíliosdoença acidentários concedidos pelos códigos da Classificação Nacional de Atividades Econômica - CNAE Classe segundo os códigos da Classificação Internacional de Doenças (10 Revisão) CID-10. Brasília, 2013b. Janeiro a maio de 2013 [tabelas].

. Ministério do Planejamento. Orçamento e Gestão. Secretaria de Gestão Pública. Boletim Estatístico de Pessoal, Brasília, v. 18, n. 211, p. 1-194, 2013c.

. Serviço Federal de Processamento de Dados - SERPRO. Sistema Siape-Saúde. Brasília, 2013d. Disponível em: < https://www2.siapenet.gov.br/saude/ Login.do?method $=\operatorname{loginSaude}>$. Acesso em: $18 \mathrm{fev}$. 2013.

. Controladoria-Geral da União. Portal da Transparência do Governo Federal: servidores. Brasília, 2013e. Disponível em: < http://www. transparencia.gov.br/>. Acesso em: 11 abr. 2013.

CAVALHEIRO, G.; TOLFO, S. R. Trabalho e depressão: um estudo com profissionais afastados do ambiente laboral. Psico-USF, Itatiba, v. 16, n. 2, p. 241-249, 2011.

CUNHA, J. C. C. B. A análise estatística dos afastamentos por problemas de saúde de servidores públicos: ferramenta para a gestão da área de saúde de servidores públicos. In: CONGRESSO CONSAD DE GESTÃO PÚBLICA, 2., 2008, Brasília. Anais eletrônicos... Brasília: CONSAD, 2008.

DEMYTTENAERE, K. et al. Prevalence, severity, and unmet need for treatment of mental disorders in the World Health Organization World Mental Health Surveys. Journal of the American Medical Association, Chicago, v. 291, n. 21, p. 2581-2590, 2004. http:// dx.doi.org/10.1001/jama.291.21.2581. PMid:15173149. 
ELOVAINIO, M. et al. Perceived organizational justice as a predictor of long-term sickness absence due to diagnosed mental disorders: results from the prospective longitudinal Finnish Public Sector Study. Social Science \& Medicine, v. 91, p. 39-47, 2013. http://dx.doi.org/10.1016/j.socscimed.2013.05.008. PMid:23849237.

FALAVIGNA, A. Prevalência e tendência temporal de afastamento do trabalho por transtornos mentais e do comportamento em enfermeiros de um hospital geral (1998-2008). 2010. 72 f. Dissertação (Mestrado em Saúde Coletiva)-Universidade Luterana do Brasil, Porto Alegre, 2010.

FARAGHER, E. B.; CASS, M.; COOPER, C. L. The relationship between job satisfaction and health: a meta-analysis. Occupational and Environmental Medicine, London, v. 62, n. 2, p. 105-112, 2005. http:// dx.doi.org/10.1136/oem.2002.006734. PMid:15657192.

GEHRING JUNIOR, G. et al. Absenteísmo-doença entre profissionais de enfermagem da rede básica do SUS Campinas. Revista Brasileira de Epidemiologia, São Paulo, v. 10, n. 3, p. 401-409, 2007. http://dx.doi. org/10.1590/S1415-790X2007000300011.

\section{INSTITUTO BRASILEIRO DE GEOGRAFIA E}

ESTATÍSTICA - IBGE. Estados@. Brasília, 2010.

Dados demográficos com base no Censo 2010.

Disponível em: <http://www.ibge.gov.br/estadosat/ index.php>. Acesso em: 26 set. 2013.

JACQUES, M. G. C.; AMAZARRAY, M. R. Trabalho bancário e saúde mental no paradigma da excelência. Boletim da Saúde, Porto Alegre, v. 20, n. 1, p. 93-105, 2006.

JUSTO, L. P.; CALIL, H. M. Depressão: o mesmo acometimento para homens e mulheres? Archives of Clinical Psychiatry, São Paulo, v. 33, n. 2, p. 74-79, 2006. http://dx.doi.org/10.1590/S010160832006000200007.

LAMY, S. et al. The organizational work factors' effect on mental health among hospital workers is mediated by perceived effort-reward imbalance: result of a longitudinal study. Journal of Occupational and Environmental Medicine, Baltimore, v. 55, n. 7, p. 809-816, 2013. http://dx.doi.org/10.1097/ JOM.0b013e31828acb19. PMid:23787570.

MARTINEZ, A. S., et al. Periódicos da CAPES: perspectiva das dissertações e teses sobre transtorno de ansiedade social/fobia social. Interação em Psicologia, Curitiba, v. 16, n. 2, p. 283-292, 2012.

MENTAL health problems in the workplace. Harvard: Harvard Health Publications, 2010. Disponível em: <http://www.health.harvard.edu/newsletters/Harvard_ Mental_Health_Letter/2010/February/mental-healthproblems-in-the-workplace>. Acesso em: 2 out. 2013.

MORRONE, L. C., et al. Saúde e Segurança do Trabalho de Servidores Públicos Estaduais: resultados iniciais de um programa em São Paulo. Revista Brasileira de Medicina do Trabalho, Belo Horizonte, v. 2, n. 2, p. 94-102, 2004.
NIEUWENHUIJSEN, K., et al. Predicting the duration of sickness absence for patients with common mental disorders in occupational health care.Scandinavian Journal of Work. Environment \& Health, Amsterdam, v. 32, n. 1, p. 67-74, 2006.

NUNES, A. V. L.; LINS, S. L. B. Servidores públicos federais: uma análise do prazer e sofrimento no trabalho. Psicologia:Organização e Trabalho, Brasília, v. 9, n. 1, p. 51-67, 2009.

ORGANIZAÇÃO MUNDIAL DA SAÚDE - OMS. Organização Pan-Americana de Saúde - OPAS. Classificação Internacional de Doenças: $10^{\mathrm{a}}$ revisão. Tradução Centro Colaborador da Organização Mundial de Saúde para a Classificação de Doenças em Português. 6. ed. São Paulo: Editora da Universidade de São Paulo, 1998. 1248p.

\section{. Relatório sobre a saúde no mundo: saúde} mental: nova concepção, nova esperança. Genebra, 2001. 135p.

PARENT-THIRION, A. et al. Fourth European Working Conditions survey. Dublin: European Foundation for the Improvement of Living and Working Conditions, 2007. 139p.

PIRES, J. C. S.; MACÊDO, K. B. Cultura organizacional em organizações públicas no Brasil. Revista de Administração Pública, Rio de Janeiro, v. 40, n. 1, p. 81-105, 2006.

SANDERSON, K.; ANDREWS, G. Common mental disorders in the workforce: recent findings from descriptive and social epidemiology. Canadian Journal of Psychiatry, Ottawa, v. 51, n. 2, p. 63-75, 2006. PMid:16989105.

SELIGMANN-SILVA, S. E. Saúde mental no trabalho contemporâneo. In: CONGRESSO INTERNACIONAL DE STRESS DA ISMA-BR, 9., 2009, Porto Alegre. Anais... Porto Alegre: ISMA-BR, 2009.

SILVA JÚNIOR, J. S. Afastamento do trabalho por transtornos mentais e fatores associados: um estudo caso-controle entre trabalhadores segurados da Previdência Social. 2012. 127 f. Dissertação (Mestrado em Saúde Pública)-Faculdade de Saúde Pública, Universidade de São Paulo, São Paulo, 2012.

TRIGO, T. R.; TENG, C. T.; HALLAK, J. E. C. Síndrome de Burnout ou Estafa Profissional e os transtornos psiquiátricos. Archives of Clinical Psychiatry, São Paulo, v. 34, n. 5, p. 223-233, 2007. http://dx.doi. org/10.1590/S0101-60832007000500004.

VEMER, P. et al. Let's get back to work: survival analysis on the return-to-work after depression. Neuropsychiatric Disease and Treatment, Albany, v. 9, p. 1637-1645, 2013. PMid:24187499.

WHITTAKER, W. et al. The effect of mental ill health on absence from work in different occupational classifications: analysis of routine data in the British Household Panel Survey. Journal of Occupational and Environmental Medicine, London, v. 54, n. 12, p. 1539-1544, 2012. http://dx.doi.org/10.1097/ JOM.0b013e3182677d12. PMid:23128300. 